\title{
Automatic 2-D/3-D Vessel Enhancement in Multiple Modality Images Using a Weighted Symmetry Filter
}

\author{
Yitian Zhao ${ }^{\circledR}$, Yalin Zheng ${ }^{\circledR}$, Yonghuai Liu, Yifan Zhao, Lingling Luo, Siyuan Yang, \\ Tong $\mathrm{Na}$, Yongtian Wang, and Jiang Liu
}

\begin{abstract}
Automated detection of vascular structures is of great importance in understanding the mechanism, diagnosis, and treatment of many vascular pathologies. However, automatic vascular detection continues to be an open issue because of difficulties posed by multiple factors, such as poor contrast, inhomogeneous backgrounds, anatomical variations, and the presence of noise during image acquisition. In this paper, we propose a novel 2-D/3-D symmetry filter to tackle these challenging issues for enhancing vessels from different imaging modalities. The proposed filter not only considers local phase features by using a quadrature filter to distinguish between lines and edges, but also uses the weighted geometric mean of the blurred and shifted responses of the quadrature filter, which allows more tolerance of vessels with irregular appearance. As a result, this filter shows a strong response to the vascular features under typical imaging conditions. Results based on eight publicly available datasets (six 2-D data sets, one 3-D data set, and one 3-D synthetic data set) demonstrate its superior performance to other state-of-the-art methods.
\end{abstract}

Index Terms-Symmetry filter, local phase, vascular, enhancement, angiography.

Manuscript received July 25, 2017; accepted September 19, 2017. Date of publication September 26, 2017; date of current version February 1, 2018. This work was supported by National Key Research and Development Program of China (2016YFB1001502), National Hi-Tech Research and Development Program (2015AA043203), National Science Foundation Program of China (61601029, 61602322, 61631010), and China Association for Science and Technology (2016QNRC001). (Corresponding author: Yitian Zhao.)

Y. Zhao is the with the Cixi Institute of Biomedical Engineering, Ningbo Institute of Industrial Technology, Chinese Academy of Sciences, Ningbo 315201, China, and also with the Beijing Engineering Research Center of Mixed Reality and Advanced Display, School of Optics and Electronics, Beijing Institute of Technology, Beijing 100081, China (e-mail: yitian.zhao@ nimte.ac.cn).

Y. Zheng is with the Department of Eye and Vision Science, University of Liverpool, Liverpool L69 3BX, U.K.

Y. Liu is with the Department of Computer Science, Aberystwyth University, Aberystwyth SY23 3FL, U.K.

Y. Zhao is with the EPSRC Centre for Innovative Manufacturing in Through-life Engineering Services, Cranfield University, Cranfield MK43 OAL, U.K.

L. Luo, S. Yang, and Y. Wang are with the Beijing Engineering Research Center of Mixed Reality and Advanced Display, School of Optics and Electronics, Beijing Institute of Technology, Beijing 100081, China.

$\mathrm{T}$. Na is with the Georgetown Preparatory School, North Bethesda, MD 20852 USA.

$\mathrm{J}$. Liu is with the Cixi Institute of Biomedical Engineering, Ningbo Institute of Industrial Technology, Chinese Academy of Sciences, Ningbo 315201, China.

Color versions of one or more of the figures in this paper are available online at http://ieeexplore.ieee.org.

Digital Object Identifier 10.1109/TMI.2017.2756073

\section{INTRODUCTION}

$\mathbf{V}$ ASCULAR diseases are among the most common public health problems worldwide. Associated conditions include diabetes, arteriosclerosis, cardiovascular diseases and hypertension, to name only the most widely occurring ones. The accurate detection and analysis of the vascular structure is essential for many clinical applications to support early detection, diagnosis, optimal treatment, and neurosurgery planning for vascular-related diseases.

Vascular imaging involves two-dimensional (2D) and threedimensional (3D) acquisition techniques for the diagnosis of different diseases. These include color fundus imaging, fluorescence angiogram (FA), magnetic resonance angiography (MRA), computed tomography angiography (CTA) and so on. Manual annotation of vascular structure is an exhausting task for graders, and computer-aided automatic/semiautomatic vascular detection methods can speed up the task significantly. However, computer-aided systems have yet to completely solve the problems as posed by the high degree of anatomical variation across the population, and the varying scales of vessels within an image. Moreover, artefacts that occur during image acquisition, such as noise, poor contrast and low resolution, exacerbate these problems. As a result, it has been proven very challenging to design a single vascular enhancement or segmentation method that will work across a variety of imaging modalities.

In general, we observe that laminar flow within blood vessels causes blood flow velocity variations, which leads to highly varying contrast distribution across different vascular structures. In consequence, we see an increase in the nonuniformity of the intensity distribution across a vascular network during image acquisition. Recent years have witnessed the rapidly increasing number of new methods for vessel segmentation from different types of medical images, as evidenced by extensive reviews, such as a general review of this topic [1] and a review of 3D vessel segmentation [2]. As blood vessels can be seen as linear structures distributed at different orientations and scales in an image, various enhancement or filtering methods have been proposed to enhance the vascular structure: to remove undesired intensity variations in the image, and to suppress non-vascular structures and image noise, thereby easing the subsequent segmentation problem [3], [4]. The most well-known intensity-based 
filtering techniques include Hessian matrix-based filters [5][7], wavelet [8], matched filters [9]-[11], flux-based [12], [13], tensor-based filtering [14], [15], Gabor filters [16], and a more recent trainable filter named the combination of shifted filter responses (COSFIRE) [17], [18].

The Hessian analysis-based image filter mainly uses the first and second order derivatives of image intensities and enables differentiation between tubular and planar structures. Several extensions have been made, such as the Weingarten matrix [19], vessel enhancement diffusion [20], and the offset medialness filter [21]. This approach has been widely used for tasks including liver investigations [22], lung vessel segmentation [23] in CTA, color fundus retinal vessel segmentation [24], coronary vasculature detection in cryomicrotome images [25], and cerebral vascular structure enhancement in MRA [7]. Wavelet-based filters are utilized for retinal vessel segmentation in [8], [24]. The wavelet-based filter is able to separate vessels from clutter and bright, localized features, as well as having the capacity to reduce noise.

The recent method proposed by Azzopardi et al. [17] considers COSFIRE [17] to detect bar-shaped structures in retinal images. This method is rotation invariant, and the orientation selectivity is determined from given vessel-like structures. It is worth noting that morphological filters such as path opening in combination with multiscale Gaussian filters have also shown some interesting results [26]. The main disadvantage of morphological methods is that they do not consider the known vessel cross-sectional shape information, and the use of an overly long structuring element may cause difficulty in detecting highly tortuous vessels [1]. Manniesing et al. reported on the feasibility of an automated post-processing tool for the segmentation of cerebral vessels from CTA using a level set method [27]. However, the use of level set method is timeconsuming for the segmentation step. Hernandez et al. [28] proposed a method based on non-parametric geodesic active regions (GAR), and provided promising segmentation results of the vasculature and aneurysms from 3D Rotational Angiography (3DRA) and CTA. Cetin and Unal introduced a cylindrical flux-based higher order tensor for vessel segmentation [14]. Quantitative validation of this method was performed on both synthetic complex tubular structures and real cerebral vasculature in MRA datasets, and in coronary arteries from CTA volumes.

Whilst intensity-based filters are susceptible to intensity inhomogeneity and will encounter further problems when they are required to faithfully enhance vessels at different scales, a local phase-based filter introduced by Lathen et al. [29] appears to be superior to intensity based filters [5], [8]-[10], [16], [24], [30] as it is resistant to intensity inhomogeneity and is capable of faithfully enhancing vessels with different widths [31], [32].

Although these aforementioned methods have been successful in achieving performance close to a human expert, there still exist many significant challenges: when the 3D retinal vascular structures with varying depths are projected onto 2D images such as retinal fundus images, it may cause overlaps of the vascular structures, poor contrast of small vessels; For both $2 \mathrm{D}$ and $3 \mathrm{D}$ vascular structure detection, most existing methods mainly focus on segmenting large vessels. It is still very challenging to extract thin vessels reliably due to imaging artifacts, poor contrast and low resolution. The same techniques might tend to over segment or miss-segment due to the varying scales of vessels within an image. As a result, it is desired to design a general enhancement method with universal parameters across a variety of image data.

By contrast of our previous work [18], in this paper, we propose a novel symmetry filter for vascular enhancement applicable to both 2D and 3D images obtained from multiple imaging modalities. It comprises two main steps. Quadratureenabled symmetry filters at different orientations at a given scale are applied to the image and the real parts representing linear structures are linearly combined and normalized to produce the response at each pixel. To make sure that the responses of the filter are invariant to structural direction, they are calculated over multiple potential orientations, combined together and normalized with their real parts being the final responses of the filter. To make sure that the responses of the filter are robust to variations in structural location and size, they are further blurred and shifted and finally maximized over neighboring pixels as a vesselness map, and the final vesselness is produced by normalizing the response from multiple scales so as to enhance vessels at different sizes. Note, the vesselness map represents the probability of a given pixel being part of a vessel.

This paper makes three contributions: i) The first contribution in theory is that the proposed method could be applied to both $2 \mathrm{D}$ and $3 \mathrm{D}$ images. In sharp contrast, most works in the literature are designed only for vessel enhancement on either 2D or 3D images. Only few methods could be applied to both 2D and 3D images such as Frangi's vesselness filter (FVF) [5], the isotropic undecimated wavelet filter (IUWF) [8], and the Local Phase Filter (LPF) [31]. These 2D/3D applicable methods (FVF, IUWF, and LPF) were selected for a comparative study in the paper and the results show that our method achieves better performance in terms of evaluation metrics such as area under the receiver operating characteristic curve (AUC), and the dice coefficient (DC); ii) The second contribution is that the proposed method has been rigorously validated quantitatively using 8 publicly accessible datasets with different imaging modalities, the results show that our proposed filter is robust to variations in the location, size and orientation of the vascular structures and the dimensionality, inhomogeneity and modality of the data; and (iii) The third contribution is that we have done manual annotations of vessels for a publicly available cerebral MRA dataset, and this annotation will be released for public access to facilitate other researchers in the community to do research and development on the same and related topics after the paper has been accepted.

\section{The Proposed Method}

In this section, we detail the proposed symmetry filter with applications to both $2 \mathrm{D}$ and $3 \mathrm{D}$ images, respectively. It includes two main steps. Firstly, we derive a symmetry filter to enhance tubular structures in a given image; Secondly, 
we blur and shift the responses of the symmetry filter and combine them with different weights at different scales and orientations so that the vascular structures at different orientations with different sizes can all be detected. The two steps, applicable to 2D images, are described in Sections II-A and II-B respectively and are then extended to 3D data in Section II-C.

\section{A. 2D Quadrature Filter}

A quadrature filter is a useful tool for extracting local structures in an image [33]. For instance, it can be used to distinguish local phase, which is an intrinsic feature invariant to changes in illumination. For a one-dimensional (1D) signal $f(x)$, the phase $\phi(x)$ at position $x$ can be simply estimated by the Hilbert transform under the concept of analytical signal such as $\phi(x)=\arctan \left(\frac{f(x)}{f_{\mathcal{H}}(x)}\right)$. However, in order to enhance spatial localization and to avoid the effects of noise, Boukerroui et al. [34] suggested that local phase should be estimated by a quadrature filter. That is,

$$
\begin{aligned}
\phi(x) & =\arctan \left(\frac{E(x)}{O(x)}\right) \\
& =\frac{f_{e}(x) * f(x)}{f_{o}(x) * f(x)},
\end{aligned}
$$

where $f_{e}(x)$ is an even (symmetric) band-pass filter while $f_{o}(x)=\mathcal{H}\left(f_{e}(x)\right)$ is the Hilbert transform of the even filter $f_{e}(x)$, as such, the filter is in quadrature.

However, in order to avoid the problems posed by the analytic signal for 2D or higher dimensions and the 2D Hilbert transform [33], a quadrature filter with even-symmetric and odd-symmetric parts is suggested by Boukerroui et al. [34]. In particular, the log-Gabor (log normal) filter is a commonly used quadrature filter [35], [36]. An oriented log-Gabor filter in the frequency domain is defined as:

$$
L G(\omega)=\exp \left(-\frac{\log ^{2}\left(\frac{\omega(p)}{\omega_{0}}\right)}{2 \log ^{2}\left(\frac{k}{\omega_{0}}\right)}\right),
$$

where the bandwidth of the filter in the radial direction can be determined by a scaling factor $k$, and $\omega_{0}$ is the center spatial frequency of the filter. $p$ is a pixel in the frequency domain, and $\omega(p)=\|p\|$. The term $\frac{k}{\omega_{0}}$ should be a constant $\left(\omega_{0}=\right.$ 0.35 in this paper) so as to achieve a constant shape-ratio filter. The responses of even and odd quadrature pair filters to an image can be estimated by:

$$
\begin{aligned}
& E(x)=\operatorname{real}\left(F^{-1}(L G(\omega) \times F(I(x)))\right), \\
& O(x)=\operatorname{imag}\left(F^{-1}(L G(\omega) \times F(I(x)))\right),
\end{aligned}
$$

where $F$ and $F^{-1}$ denote the forward and inverse Fourier transforms, respectively and $I(x)$ is the intensity of a pixel $x$ in the given image.

$O(x)$ has the maximal response at edges while $E(x)$ is almost 0 , the filter response is purely imaginary, and the filtered signal is strongly 'edge-like': while for lines, $O(x)$ is almost 0 and $E(x)$ has the maximal response. The filter response is purely real, and leads to a 'line-like' signal.
This suggests that image edges align with the zero crossing of the real part of the phase map.

In practice, multiple orientations are needed to capture structures running in different directions. Therefore, the filter response $q^{\theta}(x)$ at orientation $\theta$ is defined as:

$$
q^{\theta}(x)=E^{\theta}(x)+O^{\theta}(x) i, i=\sqrt{-1},
$$

where $E^{\theta}(x)$ and $O^{\theta}(x)$ are calculated as the sum of responses of $E(x)$ and $O(x)$ over the pixels inside a kernel centered at the pixel $x$ of interest with a size of $16 \times 16$ at an orientation of $\theta$ and normalized into the range of $[0,1]$ respectively.

The even filter is a line filter as the real part, and the odd filter is an edge filter with the imaginary part. Thus, we suggest that the real part of the response $E^{\theta}(x)$ and the absolute value of the imaginary part $O^{\theta}(x)$ are used, with a view to avoid confusion caused by changes in structural direction. Hence, the filter response $q^{\theta}(x)$ can be rewritten as $\bar{q}^{\theta}(x)=E^{\theta}(x)+\left|O^{\theta}(x)\right| i$. In order to achieve a rotationally invariant response, filters in all directions are combined as $q(x)=\sum_{\theta \in \Theta} \bar{q}^{\theta}(x)$, where $\Theta$ indicates the set of directions under consideration: $\Theta=\left\{\frac{\pi}{16}, \frac{2 \pi}{16}, \frac{3 \pi}{16}, \cdots, \frac{15 \pi}{16}, \pi\right\}$. Since the zero-crossing of the real part of $q(x)$ indicates an edge, the response $\varrho(x)$ of the quadrature filter to a pixel $x$ in a given image is defined through taking the real part of the normalized $q(x)$ as:

$$
\varrho(x)=\operatorname{real}\left\{\frac{q(x) \cdot|q(x)|}{|q(x)|^{2}+a^{2}}\right\},
$$

where $a$ is a small number $a=1 e-6$ to avoid divided by zero problem. Due to normalization, $\varrho(x) \in[0,1]$ and thus indicates the probability that a pixel lies on a vessel.

\section{B. Blurring and Shifting}

In order to allow for greater tolerance in the positions and scales of the respective contours, blurring and shifting operations are applied to the quadrature filter responses. While the blurring operation is used to suppress the noise, the shifting operation is used to enhance the response of the filter through maximizing those of neighbors in the dark and low contrast regions. As suggested by [17], we apply a Gaussian function $G_{\sigma}(u, v)$ to the responses of the proposed filter to achieve the blurring operation:

$$
\sigma=\sigma_{0}+\alpha \rho,
$$

where $\sigma$ indicates the standard deviation of the function, $\sigma_{0}$ and $\alpha$ are constants, and $\rho$ is the radius parameter representing a linear function of the distance from the center $(u, v)$ of the filter to its $11 \times 11$ neighbors. In this paper, $\sigma_{0}=2.5$ and $\alpha=0.2$. The value of $\rho$ is related to the size and complexity of the local structure.

Each blurred quadrature response $\varrho^{\prime}$ of the pixels will then be shifted respectively by a distance $\rho_{i}$ in the direction opposite to $\phi_{i}$. In Cartesian coordinates, the shift vector in question is $\left(\Delta u_{i}, \Delta v_{i}\right)$, where $\Delta u_{i}=-\rho_{i} \cos \phi_{i}$ and $\Delta v_{i}=-\rho_{i} \sin \phi_{i}$. Finally, the blurred and shifted responses $S_{\rho_{i}, \phi_{i}}$ of the quadrature filter for each tuple $\left(\rho_{i}, \phi_{i}\right)$ in the set 


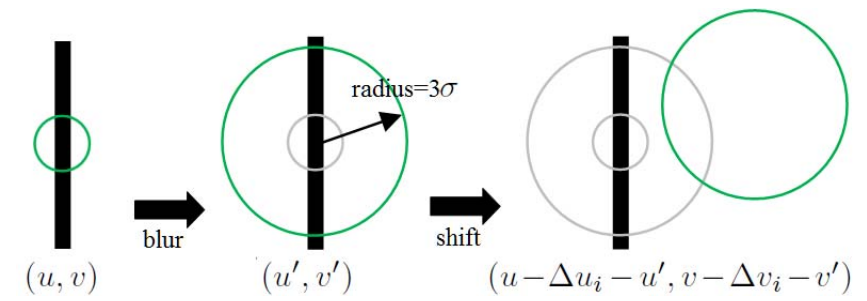

Fig. 1. The main ideas of blurring and shifting operations. (a) The initial position. (b) Gaussian blurring with a radius of $3 \sigma$. (c) Shifting specified by $\left(\rho_{i}, \phi_{i}\right)$ on the blurred response.

$S$ is defined as the maximum response $\varrho^{\prime}$ in a neighborhood of the central pixel $(u, v)$ :

$$
\begin{aligned}
& S_{\rho_{i}, \phi_{i}}(u, v) \\
& \quad=\max _{u^{\prime}, v^{\prime}}\left\{\varrho^{\prime}\left(u-\Delta u_{i}-u^{\prime}, v-\Delta v_{i}-v^{\prime}\right) G_{\sigma}\left(u^{\prime}, v^{\prime}\right)\right\},
\end{aligned}
$$

where $-3 \sigma \leq u^{\prime}, v^{\prime} \leq-3 \sigma$. The above configuration process represents a convolution of the weighting function $G_{\sigma}$ with respect to the filter center $(u, v)$, which is blurred and shifted by $\left(u-\Delta u_{i}-u^{\prime}, v-\Delta v_{i}-v^{\prime}\right)$, where the shift vector is determined by the sub-unit parameters $\left(\rho_{i}, \phi_{i}\right)$. In consequence, the shift operation is able to assemble all the responses at the proposed filter center. Fig. 1 illustrates the main ideas of the blurring and shifting operations.

The shifting operation proposed above can be denoted by a tuple $\left(\rho_{i}, \phi_{i}\right)$ of two parameters: $S=\left\{\left(\rho_{i}, \phi_{i}\right) \mid i=1, \ldots, n\right\}$, where $n$ indicates the number of quadrature responses. These parameters are able to characterize the properties of the specified area of interest. The parameter values of $S$ can be automatically determined from the aforementioned filter settings of the standard deviation of the filter responses, kernel size, and orientations: $\rho_{i} \in\{0,2,4\}$ and $\phi_{i} \in\{0,0.5 \pi, \pi, 1.5 \pi\}$. Fig. 2 shows the example responses of the original quadrature filter, and its blurring and shifting operations respectively. After these operations, the vascular structures are much clearer for visualization and interpretation.

The response $\mathcal{R}_{S}(u, v)$ of the proposed filter to a pixel at position $(u, v)$ in a given image is defined as a threshold $t$ of the power $\tau$ of a multiplication of $S_{\rho_{i}, \phi_{i}}(u, v)$ raised to the power of $\omega_{i}$ over the tuples $\left(\rho_{i}, \phi_{i}\right)$ in the set $S$ :

$$
\mathcal{R}_{S}(u, v)=\left|\left(\prod_{i=1}^{|S|}\left(S_{\rho_{i}, \phi_{i}}(u, v)^{\omega_{i}}\right)\right)^{\tau}\right|_{t},
$$

where $\tau=\frac{1}{\sum_{i=1}^{|S|} \omega_{i}}, \omega_{i}=\exp \left(-\frac{K * \rho_{i}^{2}}{2 \bar{\sigma}^{2}}\right)$, and $\bar{\sigma}=$ $\frac{1}{3} \max _{i \in\{1 \cdots|S|\}}\left\{\rho_{i}\right\}$. Here, $|\cdot|_{t}$ denotes thresholding the response at a fraction $t(0 \leq t \leq 1)$ of the maximum response. The multiplication operator is applied to ensure that a non-zero response will be achieved only when all the responses of blurred and shifted operations are greater than zero. In practice, the exponent $\omega_{i}$ achieves the maximum value $\omega_{i}=1$ when $\rho_{i}=0$, while reaching the minimum value $\omega=\exp (-K / 2)$ if $\rho_{i}=\rho_{\max } . K$ is the Gaussian parameter $(K=0.8$ in this work) that controls how heavily the $\rho_{i}$ should be penalized.
In practice, multiple scales will be needed in order to enhance vessels of different sizes in an image. The final response $\mathcal{R}(u, v)$ of the proposed filter is estimated as the weighted average of $\mathcal{R}_{S}(u, v)$ at a scale of $m$ with the weight defined as the $\beta$ th power of the magnitude of the filter response $\mathcal{R}_{S}(u, v)$ at that scale:

$$
\mathcal{R}(u, v)=\frac{\sum_{m=1}^{M} \mathcal{R}_{S}^{m}(u, v)\left|\mathcal{R}_{S}^{m}(u, v)\right|^{\beta}}{\sum_{m=1}^{M}\left|\mathcal{R}_{S}^{m}(u, v)\right|^{\beta}},
$$

where $\mathcal{R}_{S}^{m}(u, v)$ can be computed using Eq.(9) under a scale of $m$, where $m \in\{1, \cdots M\}$, and $M$ denotes the number of scales. At each scale $m$, the original given image is uniformly down-sampled to $1 /(m * m)$ of its original size. In this study, the scales used are 1 to 3 . $\beta$ is the order number of the power of the magnitude of the filter response at each scale. Fig. 3 illustrates the individual response of the proposed filter under scales 1 to 3 and the fused one, with $\beta=1$. The proposed filter is not only able to enhance the vascular structure with clear edges, but also is capable of suppressing the background in such a way as to permit vessels to be easily distinguished from the background.

\section{3D Symmetry Filter}

The quadrature filter proposed in the last two subsections is applicable to $2 \mathrm{D}$ images. In this section, we extend it to deal with 3D volumetric data by designing a 3D log-Gabor filter. This filter in the frequency domain is constructed from two components: a log-Gabor filter $L G$ and a rotationally symmetric angular Gaussian function $G$. The former filter is able to control the frequencies to which the filter responds, and the latter filter controls the orientation selectivity of the filter. Therefore, the transfer function of the 3D log-Gabor filter $\left(L G_{3}\right)$ is given as:

$$
\begin{aligned}
L G_{3} & =L G \times G \\
& \left.=\exp \left(-\frac{\log ^{2}\left(\frac{\omega(p)}{\omega_{0}}\right)}{2 \log ^{2}\left(\frac{k}{\omega_{0}}\right)}\right) \times \exp \left(-\frac{\alpha\left(p, \Phi_{1}, \Phi_{2}\right)}{2 \tau_{\alpha}^{2}}\right)\right)
\end{aligned}
$$

where $\Phi_{1}$ and $\Phi_{2}$ are the azimuth and elevation angles for the definition of the filtering sphere, $\alpha\left(p, \Phi_{1}, \Phi_{2}\right)$ is the angle $\alpha\left(p, \Phi_{1}, \Phi_{2}\right)=\arccos \left(p \cdot \frac{v}{\|p\|}\right)$ between the position vector at a point $p$ in the frequency domain and a unit vector at point on the unit sphere defined as $v=$ $\left(\sin \Phi_{1} \cos \Phi_{2}, \sin \Phi_{1} \sin \Phi_{2}, \cos \Phi_{1}\right)$, and $\tau_{\alpha}$ is the standard deviation ( $\tau_{\alpha}=0.5$ in this paper) of the Gaussian spreading function in the angular direction and describes the angular selectivity of the filter.

Similarly, the even filter $E(u, v, w)=\operatorname{real}\left(F^{-1}\left(L G_{3} \times\right.\right.$ $F(I(u, v, w))))$ and odd filter $O(u, v, w)=$ $\operatorname{imag}\left(F^{-1}\left(L G_{3} \times F(I(u, v, w))\right)\right)$ can be defined in 3D space where $F$ and $F^{-1}$ denote the forward and inverse 3D Fourier Transforms, $I(u, v, w)$ indicates the intensity value at location $(u, v, w)$. In practice, multiple orientations are again needed to capture structures at different directions, with the intention of achieving a rotationally invariant response. In $3 \mathrm{D}$, two parameters, $\theta_{1}$ and $\theta_{2}$, are needed to specify 

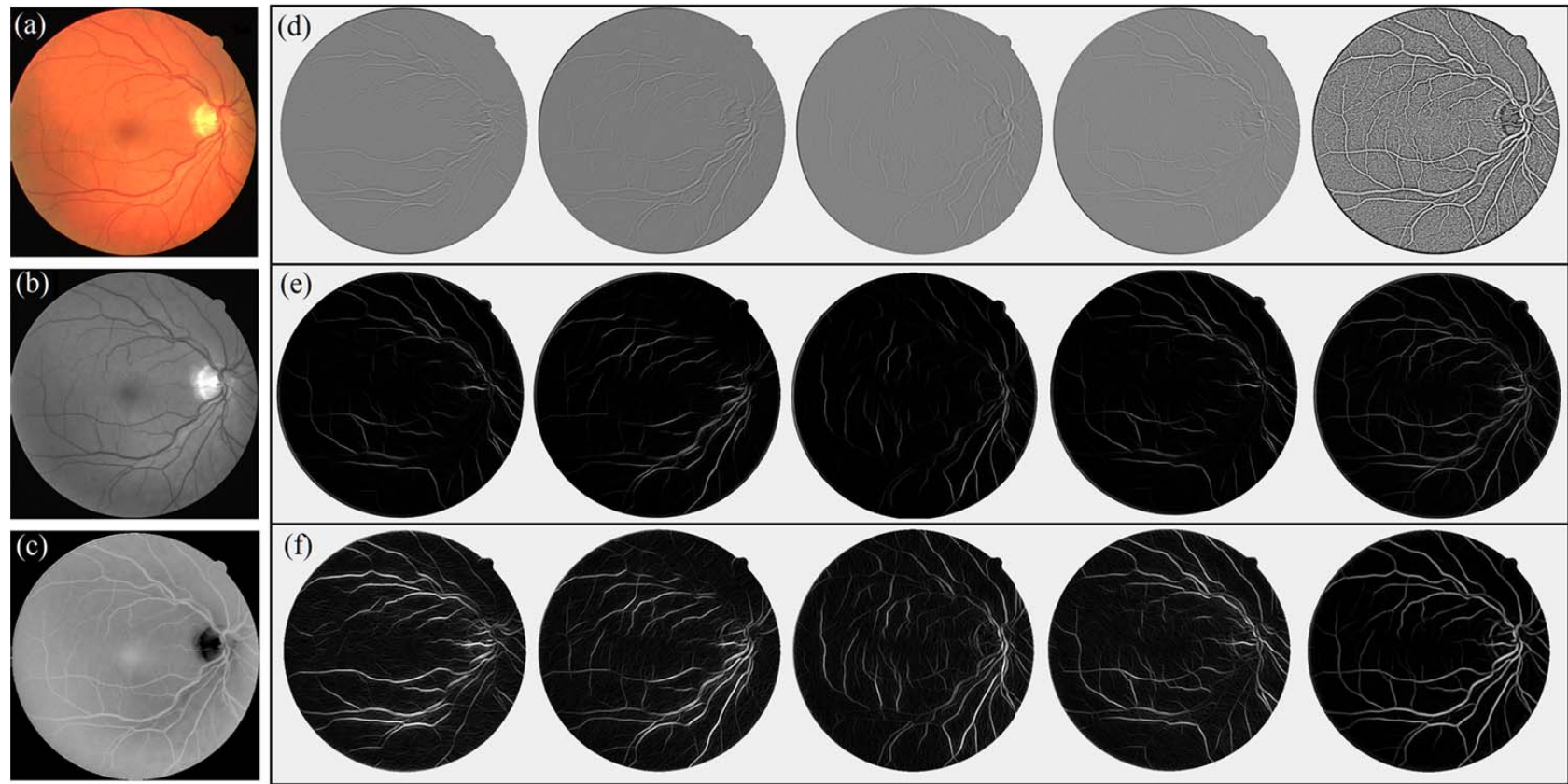

$\theta=\frac{\pi}{4}$

$\theta=\frac{\pi}{2}$

$\theta=\frac{3 \pi}{4}$

$\theta=\pi$

Fused results

Fig. 2. The responses of the original quadrature filter, its blurring and shifting operations, respectively at different orientations. (a) An example image. (b) Green channel of (a). (c) Inverted green channel of (a). (d) Responses of the original quadrature filter. (e) Responses of blurring through the Gaussian function applied to (d). (f) Responses of shifting to (e).
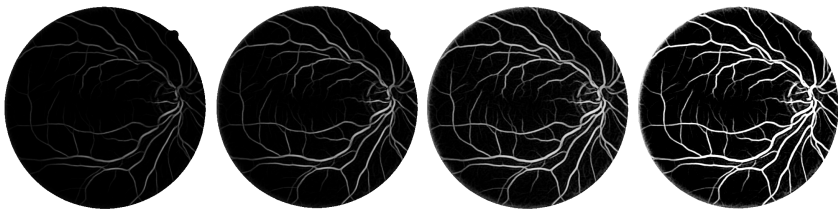

Fig. 3. The effectiveness of the responses by the proposed filter for the left to right under scales 1 to 3 , and the fused one, respectively.

the orientation of the kernel with a size of $16 \times 16 \times 16$ where, as in the 2D case, $\theta_{1} \in\left\{\frac{\pi}{16}, \frac{2 \pi}{16}, \frac{3 \pi}{16}, \cdots, \frac{15 \pi}{16}, \pi\right\}$ and $\theta_{2} \in\left\{\frac{2 \pi}{16}, \frac{4 \pi}{16}, \frac{6 \pi}{16}, \cdots, \frac{30 \pi}{16}, 2 \pi\right\}$.

Similar to the $2 \mathrm{D}$ case, the blurring and shifting operations are also useful when the symmetry filter is applied to 3D data. Therefore, Eqs. (8) and (9) can be rewritten as

$$
\begin{gathered}
S_{\rho_{i}, \phi_{i}, \gamma}(u, v, w)=\max _{u^{\prime}, v^{\prime}, w^{\prime}}\left\{\varrho \left(u-\Delta u_{i}-u^{\prime}, v-\Delta v_{i}-v^{\prime},\right.\right. \\
\\
\left.\left.w-\Delta w_{i}-w^{\prime}\right) G_{\sigma}\left(u^{\prime}, v^{\prime}, w^{\prime}\right)\right\} . \\
\mathcal{R}_{S}(u, v, w)=\left|\left(\prod_{i=1}^{|S|}\left(S_{\rho_{i}, \phi_{i}, \gamma}(u, v, w)^{\omega_{i}}\right)\right)^{\tau}\right|_{t}
\end{gathered}
$$

where the shift vector $\left(\Delta u_{i}, \Delta v_{i}, \Delta w_{i}\right)$ is defined as $\Delta u_{i}=$ $-\rho_{i} \sin \phi_{i}, \Delta v_{i}=-\rho_{i} \sin \phi_{i}, \Delta w_{i}=-\rho_{i} \tan \gamma$ and $\gamma=45^{\circ}$. Then the overall response of the proposed 3D filter is estimated as the weighted average in Eq.(10) of the responses of the filter at different scales with the weight defined as the $\beta$ th power of the magnitude of the response of the filter at each scale $m$

\section{ExPERIMENTAL RESULTS ON 2D IMAGES}

In this section, the proposed 2D symmetry filter is rigorously validated for vessel enhancement and segmentation in 2D images. A graph-cut-based model was employed to segment the vessels from the proposed enhanced vessel map [31].

\section{A. Materials}

The proposed enhancement method has been evaluated on six publicly available retinal image datasets, including four color fundus image datasets: DRIVE, ${ }^{1}$ STARE,${ }^{2}$ ARIA,${ }^{3} H R F^{4}$; one Scanning Laser Ophthalmoscopy (SLO) technique based dataset IOSTAR ${ }^{5}$; and one fluorescein angiogram (FA) dataset, VAMPIRE. ${ }^{6}$ The corresponding details of these datasets are summarized in Table I.

\section{B. Evaluation Metrics}

To compare the binary segmentation results with their corresponding manually annotated ground truths, three commonly used quantitative measures were used to quantify the performance of the competing methods in terms of pixels: sensitivity $(S E)=T P /(T P+F N)$, specificity $(S P)=$ $T N /(T N+F P)$, and accuracy $(A C C)=(T P+T N) /(T P+$ $F P+T N+F N)$ where $T P, T N, F P$ and $F N$ indicate the true positive (correctly identified vessel pixels), true negative (correctly identified background pixels), false positive (incorrectly identified vessel pixels), and false negative (incorrectly identified background pixels), respectively.

\footnotetext{
${ }^{1}$ http://www.isi.uu.nl/Research/Databases/DRIVE/

${ }^{2} \mathrm{http}: / / \mathrm{www} . c e s . c l e m s o n . e d u /$ ahoover/stare/

${ }^{3} \mathrm{http}: / / \mathrm{www}$.eyecharity.com/aria_online.html

${ }^{4}$ https://www5.cs.fau.de/research/data/fundus-images/

${ }^{5}$ http://www.retinacheck.org

${ }^{6} \mathrm{http} / / /$ vampire.computing.dundee.ac.uk/tools.html
} 
TABLE I

The Details of the Used Retinal Image Datasets, Including: the Number of Images, the Dimension of the Image, the Field of View in Degrees, the Camera Used for Data Acquisition, the IMAge Type, and the Manual AnNotation Used as Ground Truth

\begin{tabular}{|c|c|c|c|c|c|c|}
\hline Datasets & Image number & Dimension & FOV & Camera & Image type & Ground truth \\
\hline DRIVE & 40 & $565 \times 584$ & $45^{\circ}$ & Canon CR5 non-mydriatic 3-CCD & color fundus & observer 2 \\
\hline STARE & 20 & $605 \times 700$ & $35^{\circ}$ & Topcon TRV-50 & color fundus & observer 2 \\
\hline ARIA & 161 & $768 \times 576$ & $50^{\circ}$ & Zeiss FF450+ & color fundus & observer DGP \\
\hline IOSTAR & 30 & $1024 \times 1024$ & $45^{\circ}$ & EasyScan & color fundus & observer 1 \\
\hline HRF & 45 & $3504 \times 2336$ & $60^{\circ}$ & - & color fundus & observer 1 \\
\hline VAMPIRE & 8 & $3900 \times 3072$ & $200^{\circ}$ & Optos P200C & fluorescein angiogram & observer 1 \\
\hline
\end{tabular}

Since the background usually dominates and returns relatively higher SP values, a high SP value does not always mean that accurate vascular structure segmentation results have been obtained. In order to reflect the trade-offs between the sensitivity and specificity and evaluate the quality of our vascular enhancement results more reliably, AUC, G-mean (G), Dice Coefficient (DC), and $\kappa$ statistical analysis were also used. The receiver operating characteristics (ROC) curve is computed with true positive ratio (TPR) versus the false positive ratio (FPR), the G-mean is computed as $G=\sqrt{S E \times S P}$ [37]. The DC is computed as a comparison measure for determining the degree of agreement between the manual annotations and the results of the automated segmentation method. The DC is defined as $D C=2(A \cap B) /(A+B)$, where $A$ is the ground truth and $B$ indicates the segmentation result. A DC value higher than 0.70 generally indicates excellent agreement [38]. Finally, the $\kappa$ statistical analysis can measure the agreement between two raters, and is more robust than other percentage agreement measure, as it takes into account the possibility of the agreement occurring by chance - $\kappa$ values between 0.81 to 1.00 indicate almost perfect agreement, values between 0.61-0.80 exhibit substantial agreement, values of 0.41-0.60 exhibit moderate agreement and values less than 0.40 exhibit poor to fair agreement.

\section{Results}

In our experiments, the green channel of color fundus images and the original FA images were used while the originals of the other datasets were used as it is. All the experiments were carried out in MATLAB2015a on a PC with an Intel Core i7-4790K CPU, 4.00GHz, and 16GB RAM. Fig. 4 illustrates examples of vessel detection results from six datasets. The following manual vessel segmentation results were used as standard references: observer 2 of DRIVE and STARE; observer DGP of ARIA; and observer 1 of IOSTAR, HRF, and VAMPIRE.

Four other state-of-the-art vessel enhancement methods were also selected for comparison purposes: the FVF [5], IUWF [8], LPF [31] and COSFIRE [17]. For a fair comparison, their parameters were optimized for the best performance as follows:

- FVF scales: 1-8, scale ratio: 2 .

- IUWF scales: 2-3.

- LPF scales: 1-3, orientation: $\theta \in\left\{\frac{\pi i}{4} \mid i=0,1 \cdots 3\right\}$.

- COSFIRE scales: $1-4$, orientation: $\theta \in\left\{\frac{\pi i}{8} \mid i=0, \cdots 7\right\}$, threshold value: 0.35 .
TABLE ॥

Segmentation PERFormance of Using Four DifFERENT ENHANCEMENT METHODS (FVF, IUWF, LPF, COSRIRE) AND THE

Proposed Segmentation Model on Six Retinal IMAge DATASETS, IN TERMS OF SE, SP, ACC, AUC, DC AND COMPUTATION TIME $T$ (IN SECONDS)

\begin{tabular}{llllllll}
\hline \hline Dataset & Method & SE & SP & ACC & AUC & DC & $\boldsymbol{T}$ \\
\hline \multirow{5}{*}{ DRIVE } & FVF & 0.667 & 0.921 & 0.881 & 0.929 & 0.691 & 20.6 \\
& IUWF & 0.744 & 0.923 & 0.921 & 0.961 & 0.729 & 5.6 \\
& LPF & 0.744 & 0.978 & 0.953 & 0.960 & 0.782 & $\mathbf{4 . 6}$ \\
& COSFIRE & 0.766 & 0.970 & 0.944 & 0.961 & 0.777 & 10.2 \\
& Proposed & $\mathbf{0 . 7 7 4}$ & $\mathbf{0 . 9 7 9}$ & $\mathbf{0 . 9 5 8}$ & $\mathbf{0 . 9 7 5}$ & $\mathbf{0 . 7 9 3}$ & 5.5 \\
\hline \multirow{5}{*}{ STARE } & FVF & 0.754 & 0.967 & 0.938 & 0.953 & 0.651 & 18 \\
& IUWF & 0.776 & 0.954 & 0.943 & 0.953 & 0.791 & 4.5 \\
& LPF & 0.786 & 0.975 & 0.951 & 0.956 & 0.801 & $\mathbf{3 . 5}$ \\
& COSFIRE & 0.772 & 0.970 & 0.950 & 0.956 & 0.782 & 10.1 \\
& Proposed & $\mathbf{0 . 7 8 8}$ & $\mathbf{0 . 9 7 6}$ & $\mathbf{0 . 9 5 7}$ & $\mathbf{0 . 9 5 9}$ & $\mathbf{0 . 8 0 3}$ & 4.3 \\
\hline \multirow{5}{*}{ ARIA } & FVF & 0.717 & 0.934 & 0.921 & 0.948 & 0.627 & 19.7 \\
& IUWF & 0.736 & 0.920 & 0.912 & 0.951 & 0.698 & 5.6 \\
& LPF & 0.751 & 0.930 & 0.920 & 0.951 & 0.715 & $\mathbf{4 . 6}$ \\
& COSFIRE & 0.750 & 0.931 & 0.921 & 0.952 & 0.714 & 13.5 \\
& Proposed & $\mathbf{0 . 7 5 5}$ & $\mathbf{0 . 9 3 6}$ & $\mathbf{0 . 9 3 0}$ & $\mathbf{0 . 9 5 9}$ & $\mathbf{0 . 7 2 6}$ & 5.6 \\
\hline \multirow{5}{*}{ IOSTAR } & FVF & 0.704 & 0.931 & 0.920 & 0.951 & 0.688 & 58.3 \\
& IUWF & 0.726 & 0.921 & 0.911 & 0.955 & 0.709 & 21.2 \\
& LPF & 0.757 & 0.935 & 0.928 & 0.960 & 0.729 & $\mathbf{1 9 . 3}$ \\
& COSFIRE & 0.761 & 0.967 & 0.941 & 0.955 & 0.732 & 55.2 \\
& Proposed & $\mathbf{0 . 7 7 2}$ & $\mathbf{0 . 9 6 7}$ & $\mathbf{0 . 9 4 8}$ & $\mathbf{0 . 9 6 0}$ & $\mathbf{0 . 7 3 7}$ & 20.8 \\
\hline \multirow{5}{*}{ HRF } & FVF & 0.701 & 0.935 & 0.922 & 0.952 & 0.689 & 356.1 \\
& IUWF & 0.736 & 0.920 & 0.912 & 0.952 & 0.712 & 125.2 \\
& LPF & 0.751 & 0.930 & 0.920 & 0.961 & 0.733 & $\mathbf{1 1 8 . 5}$ \\
& COSFIRE & 0.744 & 0.937 & 0.932 & 0.967 & 0.738 & 320.5 \\
& Proposed & $\mathbf{0 . 7 4 9}$ & $\mathbf{0 . 9 4 2}$ & $\mathbf{0 . 9 4 1}$ & $\mathbf{0 . 9 7 1}$ & $\mathbf{0 . 7 8 6}$ & 120.3 \\
\hline \multirow{5}{*}{ VAMPIRE } & IUWF & 0.665 & 0.967 & 0.957 & 0.956 & 0.694 & 535.3 \\
& FVF & 0.708 & 0.975 & 0.966 & 0.962 & 0.711 & 187.5 \\
& COSFIRE & 0.721 & 0.984 & 0.976 & 0.964 & 0.737 & $\mathbf{1 7 0 . 3}$ \\
& Proposed & $\mathbf{0 . 7 4 1}$ & 0.985 & 0.979 & 0.967 & 0.758 & 478.2 \\
& & & & & & & \\
\hline \multirow{5}{*}{} & & & $\mathbf{0 . 9 8 1}$ & $\mathbf{0 . 9 6 9}$ & $\mathbf{0 . 7 6 4}$ & 178.5 \\
\hline & & & & & &
\end{tabular}

Figure 5 show results of applying different enhancement methods to an example image with a representative patch containing multiple vascular bifurcations (red arrows), curvature changes, intensity inhomogeneity on large vessels, and low intensities on tiny vessels (yellow arrows). It can be clearly seen that the FVF is only able to enhance vessels with larger diameters, but misses most vessels with small diameters. The IUWF also enhances background regions, which leads to difficulties in identifying the vascular structures. As for the LPF and COSFIRE, the vessel edges are clearly enhanced, and these methods achieve better results in distinguishing vessels from the background. However, the LPF also enhances the artifacts surrounded by the vessel regions, and COSFIRE yields relatively weak responses to bifurcations and crossovers and misses the small vessels. 


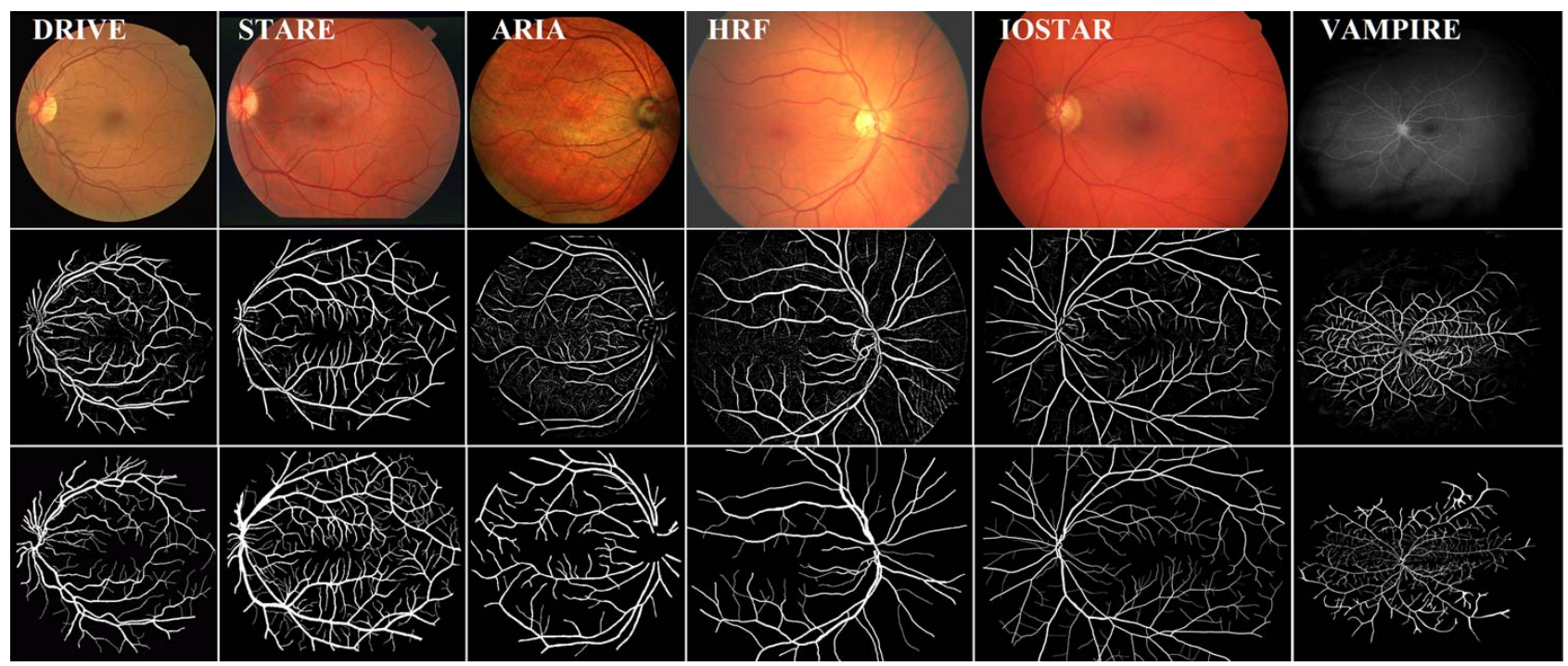

Fig. 4. Illustrative enhancement results using the proposed method on images from 6 datasets: DRIVE, STARE, ARIA, IOSTAR, HRF, and VAMPIRE, respectively. From top to bottom: original example images, enhanced results by the proposed method, and manual segmentation results.

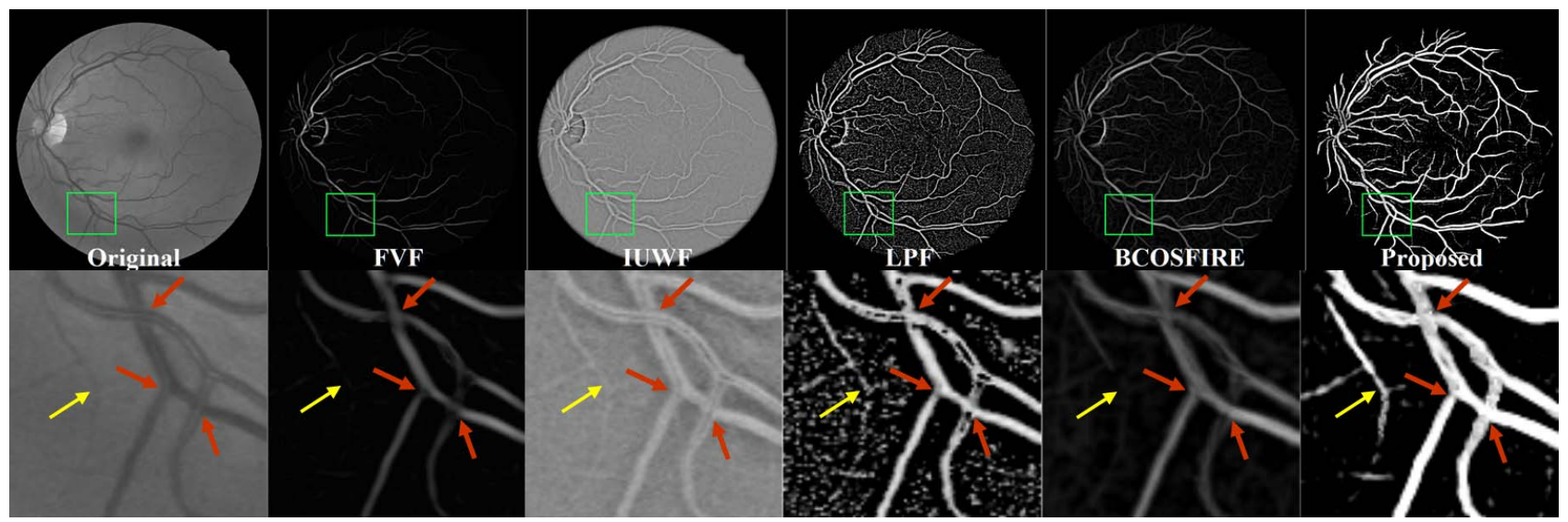

Fig. 5. Illustrative enhancement effect on a randomly chosen image from the DRIVE dataset. Top: enhancement results produced by six different vascular enhancement methods. Bottom: enhancement results on a selected region with vascular bifurcation/crossover (red arrows), and weak vessels (yellow arrow).

In sharp contrast, the proposed method is not only able to enhance the vessel regions so as to stand out more conspicuously from the background, but also has the ability to suppress noise and artifacts: stronger enhancement results on small vessels, better responses to bifurcations, and more uniform responses to both high and low intensities.

Analysis of variance (ANOVA) with Tukey post hoc analysis was performed using the SPSS version 21.0 (SPSS Inc., Chicago, IL, USA). A $p$ value of 0.05 is considered statistically significant. Statistical analysis results show that there is a statistically significant difference in SE, SP, ACC, AUC, and DC among the five filters (FVF, IUWF, LPF, COSFIRE, and the proposed method) (ANOVA, all $p<0.001$ ) over all 6 the datasets. The DC value of the proposed method $(p<0.001)$ is significantly higher than that of FVF, IUWF, LPF, or COSRIRE $(p<0.01, p<0.01, p<0.01$, and $p<0.01$ respectively).

For most vesselness filters in the literature [5], [8], [17], [31], their responses usually disconnect the bifurcations from the main vessel, and this adversely affects the sensitivity and accuracy of the final extraction of the vascular structure. The above observations are also confirmed by TABLE II.

The proposed method performs best on all the six datasets in terms of ACC, AUC and DC. In addition, the computation time of the proposed method is only slightly slower than the LPF. Additionally, as evidenced in the ROC curves in Fig. 6, the yielded results of the proposed method are very close to those of the second human observer in the DRIVE and STARE data sets (due to page limitations, the ROC curves from these two datasets are used only for illustration). Such properties are due to the proposed filter retaining the intrinsic information of features in the image that are invariant to changes in intensity, location and scale, which permit better detection of the vessels under varying conditions.

To reveal the relative performance of our proposed method, we also compared it with six existing state-ofthe-art supervised and unsupervised methods (published in the last three years) on the most popular public datasets: 
TABLE III

Performance of Different Segmentation Methods, in Terms of Se, SP, ACC, AUC, G-Mean, AND $\kappa$ ON THE DRIVE AND STARE DATASETS

\begin{tabular}{|c|c|c|c|c|c|c|c|c|c|c|c|c|}
\hline \multirow{2}{*}{ Method } & \multicolumn{5}{|c|}{ DRIVE } & & \multicolumn{5}{|c|}{ STARE } & \multirow[b]{2}{*}{$\kappa$} \\
\hline & SE & SP & $\mathrm{ACC}$ & $\mathbf{A U C}$ & G-mean & $\kappa$ & SE & SP & $\mathbf{A C C}$ & $\mathbf{A U C}$ & G-mean & \\
\hline 2nd observer & 0.776 & 0.972 & 0.947 & - & 0.868 & - & 0.895 & 0.938 & 0.934 & - & 0.916 & - \\
\hline AT & 0.740 & 0.978 & 0.949 & 0.967 & 0.859 & - & 0.732 & 0.984 & 0.956 & 0.967 & 0.849 & - \\
\hline B-COSFIRE & 0.766 & 0.970 & 0.944 & 0.961 & 0.851 & - & 0.772 & 0.970 & 0.950 & 0.949 & 0.865 & - \\
\hline IAC & 0.742 & 0.982 & 0.954 & - & 0.862 & - & 0.780 & 0.978 & 0.956 & & 0.873 & - \\
\hline LADF & 0.774 & 0.973 & 0.948 & 0.964 & 0.868 & - & 0.779 & 0.976 & 0.955 & 0.975 & 0.872 & - \\
\hline CMDT & 0.757 & 0.982 & 0.953 & 0.974 & 0.858 & - & 0.773 & 0.984 & 0.963 & 0.974 & 0.872 & - \\
\hline DT-FCCRF & 0.789 & 0.968 & - & - & 0.874 & - & 0.768 & 0.974 & - & - & 0.872 & - \\
\hline Proposed & 0.765 & 0.979 & 0.958 & 0.975 & 0.866 & 0.810 & 0.788 & 0.976 & 0.957 & 0.975 & 0.877 & $\mathbf{0 . 8 3 0}$ \\
\hline
\end{tabular}
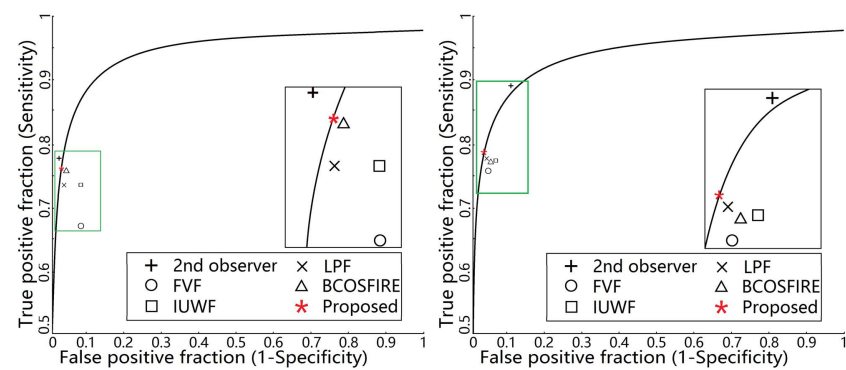

Fig. 6. ROC curves for DRIVE and STARE data sets. The results from the second observer are also reported.

DRIVE and STARE. These six methods are adaptive thresholding (AT) [39], bar-COSFIRE (B-COSFIRE) [17], infinite active contour (IAC) [32], locally adaptive derivative frame (LADF) [40], cross-modality data transformation (CMDT) [41] and discriminatively trained fully connected conditional random field (DT-FCCRF) [37]. While the last two methods are supervised, the others are unsupervised. The results are presented in TABLE III. As can be observed, the proposed method outperforms all the state-of-the-art methods on both the DRIVE and STARE datasets in terms of accuracy, and AUC. For the DRIVE dataset, the proposed method yielded a lower $\mathrm{SE}=0.774$, but higher $\mathrm{SP}=0.979$, leading to a higher score of $\mathrm{ACC}$, than the method proposed by [32]. The results of the proposed method on the STARE dataset show that its achieved SP is lower than those of the methods in [39], [32], and [41], which have sacrificed their SE score to exchange for a relatively high SP. Our method has an advantage of 0.056 in SE over the method in [39] with the highest $\mathrm{SP}=0.984$.

G-mean is capable of measuring the balance between sensitivity and specificity by taking their geometric mean, and returning a value between 0 and 1 . It can be observed that the G-mean of the proposed method is 0.882 for the STARE dataset, which achieves the best performance amongst all the methods. Good agreements were achieved for both DRIVE and STARE dataset: $\kappa=0.81$, and $\kappa=0.83$, respectively. The $\kappa$ analysis further confirms that there is a good agreement between two sets of segmentation results.

\section{EXPERIMENTAL RESULTS ON 3D DATA}

To further demonstrate the wide applicability of the proposed method for the enhancement of the data with different

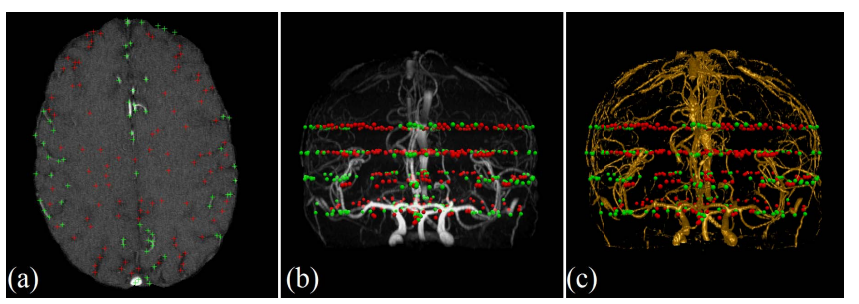

Fig. 7. Manual annotations. (a) Manually annotated landmarks displayed on one axial slice. (b) Landmarks displayed on MIP. (c) Landmarks displayed on the proposed segmentation result. Red: non-vascular region; Green: vascular region.

dimensions and modalities, we validate it over 3D data with two different imaging modalities: TOF-MRA, and synthetic images.

\section{A. Cerebral 3D TOF-MRA Images}

We first evaluate the proposed method based on a publicly available dataset - MIDAS ToF MRA images. ${ }^{7}$ The images were acquired from 25 male and 25 female healthy volunteers, aged from 18 to $60+$ years. These images were captured by a 3T MRI scanner under standardized protocols, with a voxel size of $0.5 \times 0.5 \times 0.8 \mathrm{~mm}^{3}$, and the images were reconstructed as a $448 \times 448 \times 128$ matrix. All subjects provided signed consent allowing the images to be made publicly available as open data.

In order to quantitatively evaluate the performance of the proposed method, two types of reference standard were provided in this work.

1) Manual Annotation: As in the annotations of the Grand Challenge of VESsel SEgmentation in Lung (VESSEL12) [23], we asked two graders to annotate four axial slices of each case. 1600 landmarks were labeled in each case, of which $\sim 1000$ and $\sim 600$ were annotations on vascular and non-vascular regions, respectively. The points located at the vessel bifurcations, bent vessels, or regions were annotated as vascular landmarks. Four slices which contain a high proportion of the Circle of Willis (CoW), large, and small vessels respectively were selected for annotation. A consensus between the two graders was used as the final reference standard. The manual annotation of 20 MIDASMRA images (MMM dataset) can be downloaded from the

\footnotetext{
${ }^{7}$ http://hdl.handle.net/1926/594
} 


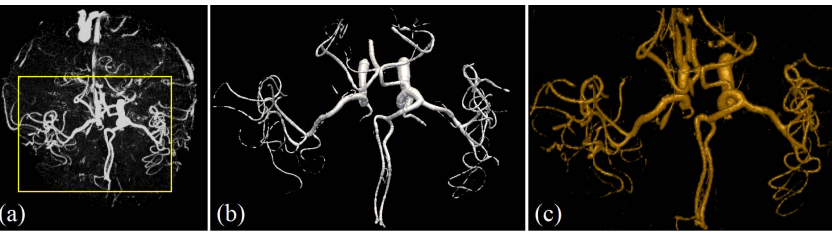

Fig. 8. Semi-manual annotations. (a) MIP view of a sample MRA: the selected region indicates the region of the CoW. (b) Semi-manual annotation on the CoW by the method of Bogunovic et al. [42]. (c) The performance of our method.

link below. ${ }^{8}$ Fig. 7 illustrates manual annotated landmarks imposed on 2D, 3D and maximum intensity projection (MIP) images, and automated segmentation results.

2) Semi-Manual Annotation: Bogunovic et al. [42] employed an open source tool kit, TubeTK [43], to generate an almostmanual annotation of the same dataset, by tracing the centerlines of the vessel, and the vessel surface was extracted using the geodesic active contour method [44], as shown as Fig. 8(b). It should be noted that this annotation concentrates on the vessels located in the region of the CoW only: accordingly, we used this annotation to validate the performance of the proposed method on it.

The top row of Fig. 9 shows an example slice from an MRA scan, and the results after having applied three state-of-the-art $3 \mathrm{D}$ vascular enhancement methods: the 3D FVF [5], the 3D IUWF [8], and the 3D LPF [29], as well as the proposed method. Their parameters were again optimized for the best performance for a fair comparison:

- FVF scales: 1-8, scale ratio: 2 .

- IUWF scales: 1-3.

- LPF scales: $2-4$, orientation: $\theta \in\left\{\frac{\pi i}{16} \mid i=0,1 \cdots 15\right\}$.

Overall, all the methods demonstrate similar performance on the large vessels. But careful observation shows that the proposed method provides relatively stronger responses to small vessels than other enhancement methods. This observation has been confirmed by the middle row of Fig. 9 - the corresponding MIP results. The proposed method provides brighter results compared to other methods and demonstrates better performance in enhancing the small vessels indicated by the green arrow at the bottom right of the figures. The bottom row of Fig. 9 shows the segmentation results obtained using different methods: visually, it reveals that our method has successfully detected more small vessels.

As it is difficult to demonstrate conclusively the superiority of the proposed method over other enhancement methods purely by visual inspection, a more objective evaluation was undertaken. First, Table IV shows the results of the obtained filter responses performed on the manual annotation, across over 50 cerebral MRA images, in terms of TP, FN, FP, and DC. Overall, the proposed method achieves the best scores in each of the metrics, with $\mathrm{TP}=95.78 \%, \mathrm{FN}=4.42 \%$, $\mathrm{FP}=6.62 \%$, and $\mathrm{DC}=95.01 \%$. In order to assess vessel detection performance over different diameters/levels/scales, the segmentation results with regard to different levels and sizes of vessels are also presented. The evaluation scores of

\footnotetext{
${ }^{8}$ http://imed.nimte.ac.cn/en-resources.html
}

TABLE IV

Segmentation Performance of Three DifFerent LeVELS OF Vessels: Small Vessels, Large Vessels, CoW, and OVerall PERFORMANCE IN TERMS OF TP, FN, FP, DC, AND RUNNING TIME $T$ (IN MINUTES)

\begin{tabular}{|c|c|c|c|c|c|c|c|}
\hline Levels & $\overline{\text { Methods }}$ & \multirow{2}{*}{\multicolumn{2}{|c|}{$\frac{\mathbf{T P}(\%)}{0554}$}} & $\overline{\mathbf{F N}(\%)}$ & $\overline{\mathbf{F P}(\%)}$ & $\overline{\overline{\mathbf{D C}}(\%)}$ & $\overline{\bar{T}}$ \\
\hline \multirow{4}{*}{ CoW } & FVF & & & 4.37 & 6.48 & 94.91 & - \\
\hline & IUWF & \multicolumn{2}{|c|}{95.68} & 4.18 & 6.32 & 95.14 & - \\
\hline & LPF & \multicolumn{2}{|c|}{95.82} & 4.12 & 6.11 & 95.29 & - \\
\hline & Proposed & \multicolumn{2}{|c|}{96.23} & 3.91 & 5.98 & 95.63 & \\
\hline \multirow{4}{*}{ Large } & FVF & \multicolumn{2}{|c|}{95.56} & 5.04 & 6.85 & 94.84 & - \\
\hline & IUWF & \multicolumn{2}{|c|}{95.71} & 4.82 & 6.42 & 95.04 & - \\
\hline & LPF & \multicolumn{2}{|c|}{95.82} & 4.65 & 6.41 & 95.19 & - \\
\hline & Proposed & \multicolumn{2}{|c|}{96.01} & 4.34 & 6.22 & 95.52 & - \\
\hline \multirow{4}{*}{ Small } & FVF & \multicolumn{2}{|c|}{82.23} & 8.24 & 7.48 & 83.45 & - \\
\hline & IUWF & \multicolumn{2}{|c|}{87.27} & 5.02 & 8.13 & 88.24 & - \\
\hline & LPF & \multicolumn{2}{|c|}{89.31} & 6.47 & 7.71 & 89.74 & - \\
\hline & Proposed & \multicolumn{2}{|c|}{92.72} & 5.65 & 7.12 & 92.01 & - \\
\hline \multirow{4}{*}{ Overall } & FVF & \multicolumn{2}{|c|}{91.43} & 5.24 & 6.98 & 91.41 & 10.8 \\
\hline & IUWF & \multirow{2}{*}{\multicolumn{2}{|c|}{93.80}} & 5.02 & 7.02 & 92.44 & 2.3 \\
\hline & LPF & & & 4.83 & 6.91 & 93.89 & 1.8 \\
\hline & Proposed & \multicolumn{2}{|c|}{95.78} & 4.42 & 6.62 & 95.01 & 2.0 \\
\hline \multicolumn{8}{|c|}{ TABLE V } \\
\hline \multicolumn{8}{|c|}{ Segmentation Results Obtained on the Circle of } \\
\hline \multirow{2}{*}{\multicolumn{8}{|c|}{$\begin{array}{l}\text { WILLIS BY USING DIFFERENT CEREBRAL VASCULAR } \\
\text { SEGMENTATION METHODS }\end{array}$}} \\
\hline & & & & & & & \\
\hline Methods & $\mathbf{T P}(\%$ & & & $\mathbf{N}(\%)$ & $\overline{\mathbf{F P}(\%)}$ & & $(\%)$ \\
\hline HOOI & $94.54 \pm$ & & & $5 \pm 0.68$ & $7.45 \pm 2.98$ & 92.8 & \pm 3.45 \\
\hline RAnk-1 & $95.43 \pm$ & & & $7 \pm 0.61$ & $6.57 \pm 1.93$ & 94.7 & \pm 2.89 \\
\hline ITM & $93.07 \pm$ & & & $3 \pm 1.41$ & $9.82 \pm 2.91$ & 91.7 & \pm 3.93 \\
\hline Proposed & $96.24 \pm$ & & & $7 \pm 0.61$ & $5.91 \pm 1.32$ & 95.5 & 2.87 \\
\hline Inter-Obs & $98.74 \pm$ & & & $3 \pm 0.57$ & $3.98 \pm 1.22$ & 98.3 & \pm 2.23 \\
\hline
\end{tabular}

our method are slightly higher at the level of the CoW and large vessels. However, on small vessels, our method offers significant improvements over the other three enhancement methods by $10.49 \%, 5,35 \%$ and $3.41 \%$ in TP respectively. Statistical analysis results show that there is a statistically significant difference in TP, FN, FP, and DC among the four filters (FVF, IUWF, LPF, and the proposed method) (ANOVA, all $\mathrm{p}<0.005)$ for three different levels of vessels, and overall performance. The DC value of the proposed method $(p<0.005)$ is significantly higher than that of the FVF, IUWF, or LPF ( $p<0.02, p<0.01$, and $p<0.01$ respectively).

In addition, evaluations of performance on segmenting the CoW were obtained based on semi-manual annotation provided by Bogunovic et al. [42], and compared with three state-of-the-art cerebral vessel segmentation methods: Higher Order Orthogonal Iteration (HOOI) [14], Finding Rank1Approximation (RAnk-1 Approx) [15], and Intensity-based Tensor Model (ITM) [13], as shown in TABLE V. The results show that our method achieves results competitive with those obtained by inter-observer manual annotation, and achieves the best overall performance among these methods, with $\mathrm{TP}=$ $96.24 \pm 0.88 \%, \mathrm{FN}=3.87 \pm 0.61 \%, \mathrm{FP}=5.91 \pm 1.32 \%$, and $\mathrm{DC}=95.53 \pm 2.87 \%$ respectively.

\section{B. Synthetic Vascular Images}

We also evaluate the proposed vessel enhancement method on $3 \mathrm{D}$ synthetic vascular images with known ground truth: 


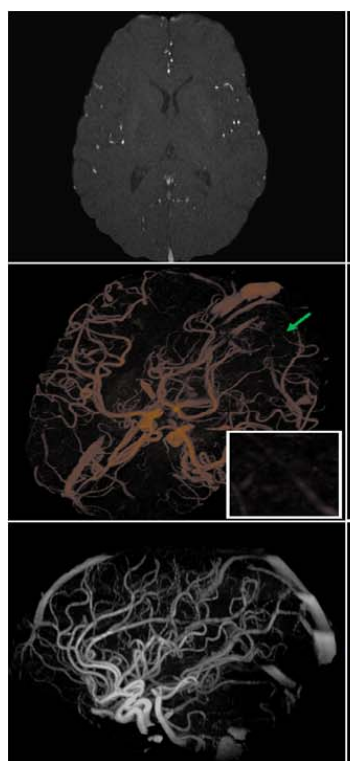

(a) Original

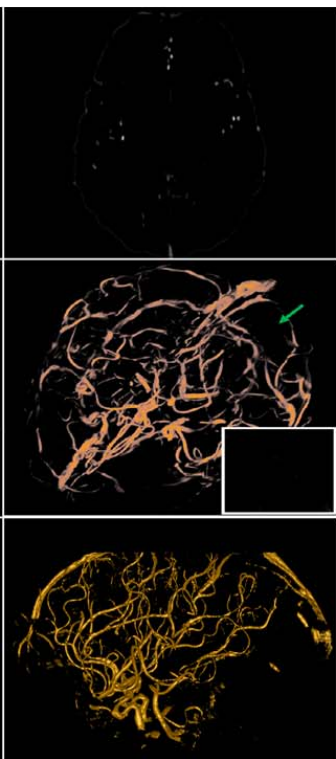

(b) FVF

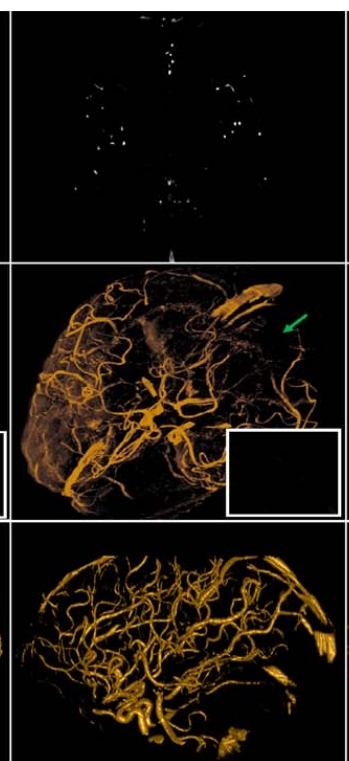

(c) IUWF

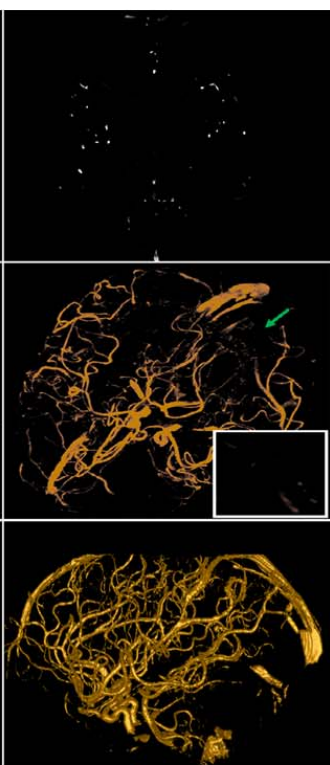

(d) LPF

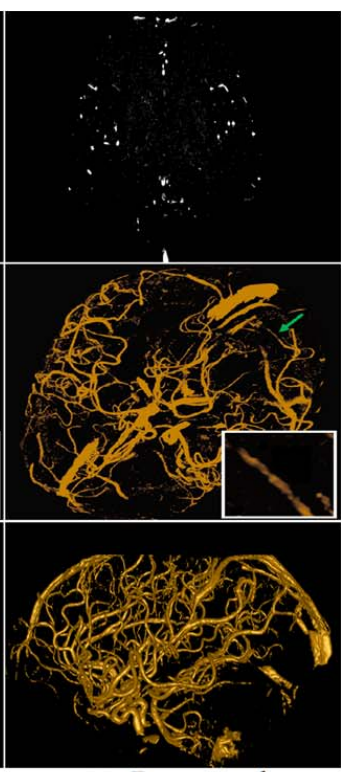

(e) Proposed

Fig. 9. Performances of different vessel detection methods on both 2D and 3D images. From top to bottom: enhancement results on one axial slice; enhanced results visualized in 3D by MIP; vascular segmentation results.

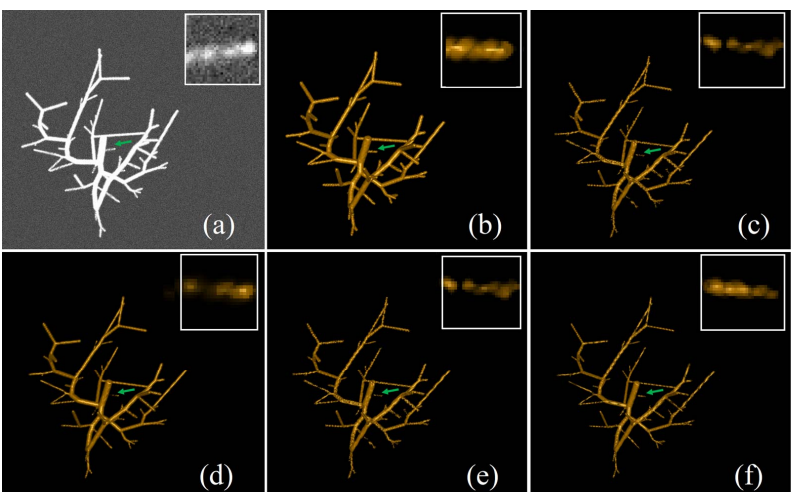

Fig. 10. Segmented vascular structure by different enhancement methods over the VascuSynth dataset 1, and the original input data (a) was corrupted by Gaussian noise of $\sigma^{2}=60$ and displayed in MIP view. (b) Ground truth. (c) FVF. (d) IUWF. (e) LPF. (f) Proposed method.

the VascuSynth vascular database [45]. This dataset aims to provide an abundance of 3D images for automated analysis of tree-like structures, which include vessel segmentation and detection of bifurcation points using the VascuSynth Software [46]. It simulates volumetric images (volume size $10 \times 100 \times 100$ voxels) of vascular trees and generates the corresponding ground truth segmentation, bifurcation locations, branch properties, and tree hierarchy. As in the previous case of cerebral vessel segmentation, the evaluation metrics TP, FN, $\mathrm{FP}$, and DC were used for validation.

In order to demonstrate the superiority of the proposed filter with application to 3D segmentation, Gaussian noise with a standard variance $\left(\sigma^{2}=60\right)$ was added here to mimic imaging artifacts, as shown in Fig. 10. It shows an example MIP view from VascuSynth and the comparative results of four filters: FVF, IUWT, LPF, and the proposed filter. Overall, all methods are capable of detecting large vessels. However,
TABLE VI

Segmentation Performance on the Synthetic Images by DifFERENT METHODS IN TERMS OF TP, FN, FP, DC, AND RUNNING TIME T IN MINUTES

\begin{tabular}{lccccc}
\hline \hline Methods & TP(\%) & FN(\%) & FP(\%) & DC(\%) & T \\
\hline FVF & $91.43 \pm 2.87$ & $4.24 \pm 2.01$ & $6.48 \pm 1.88$ & $91.41 \pm 1.76$ & 5.6 \\
IUWF & $93.87 \pm 1.93$ & $4.02 \pm 1.79$ & $6.02 \pm 1.78$ & $93.44 \pm 1.49$ & $\mathbf{0 . 8}$ \\
LPF & $95.12 \pm 1.67$ & $3.83 \pm 1.54$ & $5.91 \pm 1.21$ & $94.89 \pm 1.21$ & 1.2 \\
Proposed & $\mathbf{9 6 . 7 8} \pm \mathbf{1 . 1 3}$ & $\mathbf{3 . 4 2} \pm \mathbf{1 . 0 9}$ & $\mathbf{5 . 6 2} \pm \mathbf{1 . 2 8}$ & $\mathbf{9 6 . 0 1} \pm \mathbf{1 . 1 9}$ & $\mathbf{0 . 8}$ \\
\hline
\end{tabular}

careful observation at the location indicated by the green arrow shows that the proposed method has an advantage in enhancing the small vessels and the vessels in the dark and low contrast regions. This observation is confirmed by the results in TABLE VI- the proposed method achieves the best performance in terms of TP, FN, FP, and DC. Statistical analysis shows that there is a statistically significant difference in TP, FN, FP, and DC among four filters (FVF, IUWF, LPF, and the proposed method) (ANOVA, all $\mathrm{p}<0.002$ ). The DC value of the proposed method $(p<0.005)$ is significantly higher than that of the FVF, IUWF, or LPF $(p<0.02$, $p<0.01$, and $p<0.008$ respectively).

TABLE VII depicts the comparison of our method and the other state-of-the-art segmentation methods. For simplicity, Intensity based Tensor Model (ITM) [13] and Constrained $B$-Snake (CBS) [47] were selected for the comparative study, because they also reported the evaluation results over the data under varying levels of noise corruption $\left(\sigma^{2}=20, \sigma^{2}=60\right)$. The superiority in the scores of the proposed method over the other methods can be observed in all measures - our method outperforms the two competitors at both low and high noise levels, showing that it is resistant to imaging noise for vascular structure segmentation. 
TABLE VII

Performance of Three Different Segmentation Methods on the Synthetic Dataset in the PRESEnCE of TWO LEVEls of GAUSSIAN NoISE: $\sigma_{1}=\sigma^{2}=20, \sigma_{2}=\sigma^{2}=60$

\begin{tabular}{ll|cccccc}
\hline \hline \multirow{2}{*}{} & & \multicolumn{2}{|c}{ Data 1 } & \multicolumn{2}{c}{ Data 2 } & \multicolumn{2}{c}{ Data 3 } \\
\cline { 3 - 8 } & & $\sigma_{1}$ & $\sigma_{2}$ & $\sigma_{1}$ & $\sigma_{2}$ & $\sigma_{1}$ & $\sigma_{2}$ \\
\hline \multirow{2}{*}{$\mathbf{T P}(\%)$} & ITM & 92.89 & 90.65 & 93.78 & 91.43 & 94.23 & 92.28 \\
& CBS & 94.38 & 90.73 & 94.87 & 91.27 & 95.29 & 93.08 \\
& Proposed & $\mathbf{9 5 . 2 3}$ & $\mathbf{9 1 . 1 3}$ & $\mathbf{9 5 . 4 7}$ & $\mathbf{9 1 . 9 8}$ & $\mathbf{9 6 . 0 3}$ & $\mathbf{9 3 . 9 8}$ \\
\hline \multirow{3}{*}{$\mathbf{F N}(\%)$} & ITM & 7.11 & 9.35 & 6.22 & 8.57 & 5.77 & 7.72 \\
& CBS & 5.62 & 9.27 & 5.13 & 8.73 & 4.71 & 6.92 \\
& Proposed & $\mathbf{5 . 2 3}$ & $\mathbf{8 . 9 7}$ & $\mathbf{4 . 6 8}$ & $\mathbf{8 . 1 9}$ & $\mathbf{4 . 5 1}$ & $\mathbf{6 . 6 7}$ \\
\hline \multirow{2}{*}{$\mathbf{F P}(\%)$} & ITM & 8.45 & 8.97 & 5.13 & 8.73 & $\mathbf{4 . 7 1}$ & 6.92 \\
& CBS & 7.49 & 8.93 & 5.38 & 8.07 & 5.63 & 6.78 \\
& Proposed & $\mathbf{7 . 2 8}$ & $\mathbf{8 . 6 5}$ & $\mathbf{5 . 0 1}$ & $\mathbf{7 . 8 6}$ & 5.26 & $\mathbf{6 . 3 9}$ \\
\hline \multirow{3}{*}{$\mathbf{D C}(\%)$} & ITM & 92.27 & 90.82 & 93.54 & $\mathbf{9 1 . 7 3}$ & 94.06 & 92.76 \\
& CBS & 93.83 & 90.68 & 94.55 & 91.18 & 94.89 & 92.88 \\
& Proposed & $\mathbf{9 4 . 1 3}$ & $\mathbf{9 1 . 2 3}$ & $\mathbf{9 5 . 0 1}$ & 91.68 & $\mathbf{9 5 . 4 3}$ & $\mathbf{9 3 . 3 8}$ \\
\hline \hline
\end{tabular}

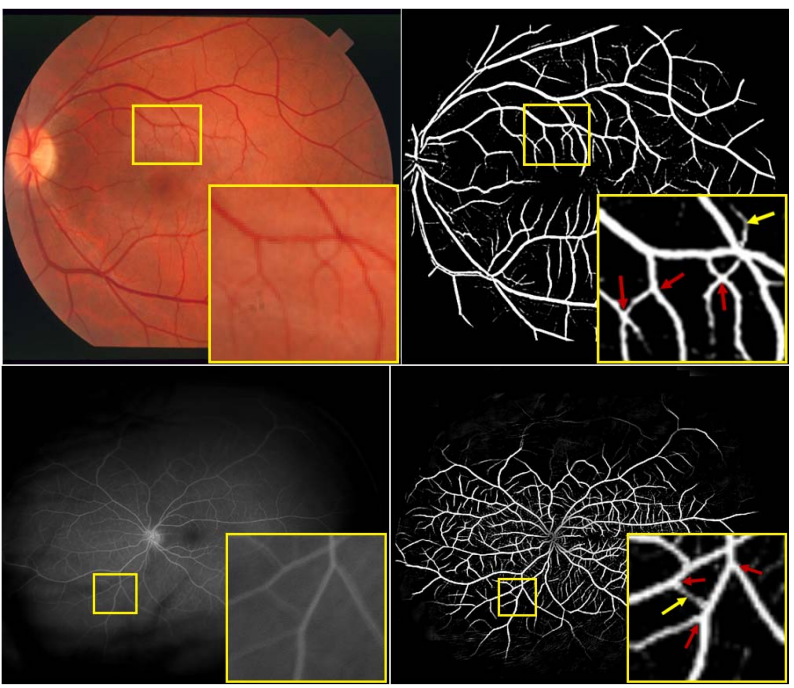

Fig. 11. Enhancement performance in the presence of weak vessels and vessel bifurcation / crossover.

\section{Discussion AND CONCLUSIONS}

In general, bifurcation and crossover of vessels, small vessels and highly curved vessels are the most challenging ones in retinal vessel segmentation. In this work, to detect small vessels, we exploited the advantages of a quadrature and symmetry filter, which retains the intrinsic information of features in the image that are invariant to changes in amplitude and phase, as a way of dealing with intensity inhomogeneity. The multi-scale approach was also used to handle the wide range of vessel calibers. Furthermore, the filter output was then calculated as the product of the blurred and shifted quadrature filter responses, so as to resolve the weak response to vessel bifurcation and crossover yielded by the most filter-based methods. It can be observed in Fig. 11 that the proposed method is able to preserve most of the small vessel structures (yellow arrows), and additionally that strong responses are achieved at bifurcations and in crossover regions (red arrows).

A further major advantage of the proposed work is that it is well-suited to $3 \mathrm{D}$ medical image data. The proposed method has been tested on MRA, and noise-degraded synthetic images. It is worth noting that we have also released a manual annotation on cerebral 3D TOF-MRA images for vessel segmentation - MMM dataset. The landmarks were not only graded from vascular structures, such as bifurcations and highly curved vessels, but were also classified into groups based on vessel diameters, to provide clinicians with more information about abnormalities at different levels of vessel segments. A fine segmentation is extremely desirable for diagnostics and surgery, e.g., embolization surgical planning. Such surgery requires accurate estimation of the geometry and volume of vessels/aneurysms. For instance, the CoW is known to be the most common site of pathologies, its rupture or leakage can result in a sub-arachnoid hemorrhagic stroke. Therefore, in order to assess the vessel detection performance of the framework over different diameters/levels/scales, the slices which contain a high proportion of the CoW, large, and small vessels were selected for annotation.

In addition, we followed Bogunovic's strategy [42], [44], and extended the semi-manual annotation to the entire vascular network, rather than the CoW only. Fig. 12 depicts the visual comparison of two reference standards (manual and semimanual annotation) with automated segmentation results and an MIP view of an example MRA scan. Fig. 12 (a) shows the manual annotated landmarks superimposed on an MIP view: the green dots indicate the vessel areas while the red dots indicate the non-vessel regions. Fig. 12 (b) shows the manually annotated landmarks plotted on the semi-manual annotated vascular structure. Fig. 12 (c) compares the results of manual annotation and that automated by the proposed method. Fig. 12 (d) contrasts the semi-manual annotated vascular structure with the proposed automated segmentation method. It can be seen from Fig. 12 (b) that many thin vessels are unannotated, because the semi-manual annotation of vessels was detected by the geodesic active contour model [42], and this model yields relatively poor performance in dark regions. Fig. 12 (c) and (d) reveal the superior performance of the proposed method: the final segmentation reveals many vessels, as the vessel structure in the low-contrast and dark regions have also been successfully detected.

The thresholding parameter $t$ in Eq.(9) controls the final responses of the proposed filter. In this case, there is a concern that it might affect the final filtering results of vessel segmentation. To address this concern, we experimentally investigate its setup - by varying the threshold $t$ from 0 to 1 in increments of 0.05 , as shown in Fig. 13. (Left: the average AUC performance on six retinal image datasets with standard deviation. Right: the average DC performance on 3D cerebral MRA and synthetic images with standard deviation.) The optimal values, $t=0.75$ and $t=0.70$ yielded the best performance in $2 \mathrm{D}$ and $3 \mathrm{D}$ data, respectively.

In conclusion, we have described and evaluated our new filter-based technique for vessel enhancement and detection for both 2D and 3D images. By means of a quadrature and symmetry filter combined with blurring and shifting operation, quantitative evaluations on publicly-accessible datasets show that, compared to the state-of-the-art methods, the proposed filter achieves better vessel segmentation results, especially 


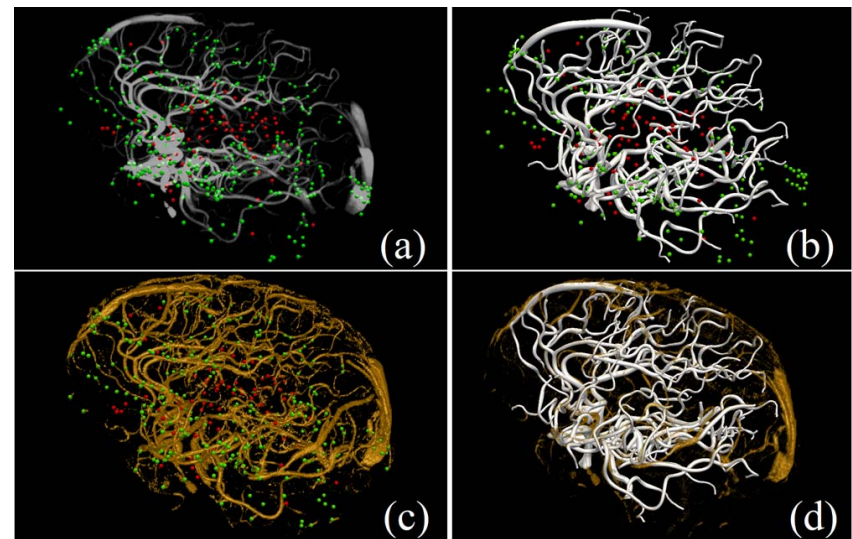

Fig. 12. Comparison of (a) MIP view and manual annotation; (b) Manual and semi-manual annotation; (c) Manual annotation and automated segmentation; (d) Semi-manual annotation and automated segmentation.
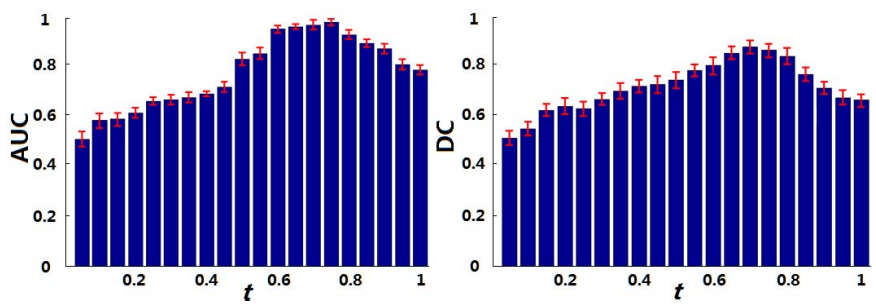

Fig. 13. The AUC and DC value of the proposed method with the threshold $t$ taking different values in 2D and 3D data, respectively.

in the dark and low contrast regions. There has been an increasing use of 3D images in clinical settings, we foresee that the proposed framework will be readily extended to other 3D images, such as Digital Subtraction Angiography (DSA). We expect that this framework will become a powerful tool in the analysis of vasculatures for the management of a wide spectrum of vascular-related diseases.

\section{ACKNOWLEDGMENT}

The authors would like to thank Professor Gozde Unal from Istanbul Technical University for her valuable suggestions, and Dr. Hrvoje Bogunovic from Medical University of Vienna for sharing the semi-manual segmentation results of 50 subjects in the MIDAS MRA dataset.

\section{REFERENCES}

[1] M. Fraz et al., "Blood vessel segmentation methodologies in retinal images-A survey," Comput. Meth. Prog. Bio., vol. 108, no. 1, pp. 407-433, 2012.

[2] D. Lesage, E. D. Angelini, I. Bloch, and G. Funka-Lea, "A review of 3D vessel lumen segmentation techniques: Models, features and extraction schemes," Med. Image Anal., vol. 13, no. 6, pp. 819-845, Aug. 2009.

[3] Y. Chen et al., "Curve-like structure extraction using minimal path propagation with backtracking," IEEE Trans. Image Process., vol. 25, no. 2, pp. 988-1003, Feb. 2016.

[4] G. Yang, Y. Chen, X. Ning, Q. Sun, J. L. Coatrieux, and H. Shu, "Automatic coronary calcium scoring using noncontrast and contrast CT images," Med. Phys., vol. 43, no. 5, pp. 2174-2186, 2016.

[5] A. F. Frangi, W. J. Niessen, K. L. Vincken, and M. V. Viergever, "Multiscale vessel enhancement filtering," in Proc. Med. Image Comput. Comput. Assist. Intervent., 1998, pp. 130-137.

[6] Y. Sato, C. Westin, A. Bhalerao, S. Nakajima, N. Shiraga, and S. Tamura, "Tissue classification based on 3D local intensity structure for volume rendering, "IEEE Trans. Vis. Comput. Graph., vol. 6, no. 2, pp. 160-180, Apr. 2000.
[7] T. Jerman, F. Penus, B. Likar, and Z. Spiclin, "Enhancement of vascular structures in 3D and 2D angiographic images," IEEE Trans. Med. Imag. vol. 35, no. 9, pp. 2107-2118, Sep. 2016.

[8] P. Bankhead, J. McGeown, and T. Curtis, "Fast retinal vessel detection and measurement using wavelets and edge location refinement," PLoS ONE, vol. 7, p. e32435, Jun. 2009.

[9] B. Zhang, L. Zhang, L. Zhang, and F. Karray, "Retinal vessel extraction by matched filter with first-order derivative of Gaussian," Comput. Biol. Med., vol. 40, no. 4, pp. 438-445, 2010.

[10] H. Narasimha-Iyer, V. Mahadevan, J. M. Beach, and B. Roysam, "Improved detection of the central reflex in retinal vessels using a generalized dual-Gaussian model and robust hypothesis testing," IEEE Trans. Inf. Technol. Biomed., vol. 12, no. 3, pp. 406-410, May 2008.

[11] Y. Zhao et al., "Saliency driven vasculature segmentation with infinite perimeter active contour model," Neurocomputing, vol. 259, pp. 201-209, Aug. 2017.

[12] D. Lesage, E. D. Angelini, I. Bloch, and G. Funka-Lea, "Bayesian maximal paths for coronary artery segmentation from 3D CT angiograms," in Proc. Med. Image Comput. Comput. Assist. Intervent., 2009, pp. 222-229.

[13] S. Cetin, A. Demir, A. Yezzi, M. Degertekin, and G. Unal, "Vessel tractography using an intensity based tensor model with branch detection," IEEE Trans. Med. Imag., vol. 32, no. 2, pp. 348-363, Feb. 2013.

[14] S. Cetin and G. Unal, "A higher-order tensor vessel tractography for segmentation of vascular structures," IEEE Trans. Med. Imag., vol. 34, no. 10, pp. 2172-2185, Nov. 2015.

[15] T. Schultz and H. P. Seidel, "Estimating crossing fibers: A tensor decomposition approach," IEEE Trans. Vis. Comput. Graphics, vol. 14, no. 6, pp. 1635-1642, Nov. 2008.

[16] J. V. B. Soares, J. J. G. Leandro, R. M. Cesar, H. F. Jelinek, and M. J. Cree, "Retinal vessel segmentation using the 2-D Gabor wavelet and supervised classification," IEEE Trans. Med. Imag., vol. 25, no. 9, pp. 1214-1222, Sep. 2006.

[17] G. Azzopardi, N. Strisciuglio, M. Vento, and N. Petkov, "Trainable COSFIRE filters for vessel delineation with application to retinal images," Med. Image Anal., vol. 19, pp. 46-57, Sep. 2015.

[18] L. Luo, Y. Zhao, J. Yang, Y. Zheng, D. Ai, and Y. Wang, "Multiscale vessel enhancement filtering," in Proc. Int. Symp. Biomed. Imag., 2017, pp. 942-946.

[19] R. Manniesing, M. A. Viergever, and W. J. Niessen, "Vessel enhancing diffusion: A scale space representation of vessel structures," Med. Image Anal., vol. 10, no. 6, pp. 815-825, 2006.

[20] M. Jiang, Q. Ji, and B. McEwen, "Model-based automated extraction of microtubules from electron tomography volume," IEEE Trans. Inf. Technol. Biomed., vol. 10, no. 3, pp. 608-617, Mar. 2006.

[21] K. Krissian, G. Malandain, N. Ayache, R. Vaillant, and Y. Trousset, "Model-based detection of tubular structures in 3D images," Comput. Vis. Image Understand., vol. 80, no. 2, pp. 130-171, 2000.

[22] H. Luu, C. Klink, A. Moelker, W. Niessen, and T. Walsum, "Quantitative evaluation of noise reduction and vesselness filters for liver vessel segmentation on abdominal CTA images," Phys. Med. Biol., vol. 60, no. 10 , pp. 3905-3926, 2015.

[23] R. Rudyanto et al., "Comparing algorithms for automated vessel segmentation in computed tomography scans of the lung: The VESSEL12 study," Med. Image Anal., vol. 18, pp. 1217-1232, Sep. 2014.

[24] Y. Wang, G. Ji, P. Lin, and E. Trucco, "Retinal vessel segmentation using multiwavelet kernels and multiscale hierarchical decomposition,' Pattern Recognit., vol. 46, pp. 2117-2133, Apr. 2013.

[25] A. Goyal, J. Lee, P. Lamata, J. Wijngaard, and P. Horssen, "Modelbased vasculature extraction from optical fluorescence cryomicrotome images," IEEE Trans. Med. Imag., vol. 32, no. 1, pp. 56-72, Jan. 2013.

[26] F. Rossant, M. Badellino, A. Chavillon, I. Bloch, and M. Paques, "A morphological approach for vessel segmentation in eye fundus images, with quantitative evaluation," J. Med. Imag. Health Informat., vol. 1, no. 1, pp. 42-49, 2011.

[27] R. Manniesing, M. Viergever, A. Lugt, and W. Niessen, "Cerebral arteries: Fully automated segmentation from CT angiography: A feasibility study," Radiology, vol. 247, pp. 247-846, Aug. 2008.

[28] M. Hernandez and A. F. Frangi, "Non-parametric geodesic active regions: Method and evaluation for cerebral aneurysms segmentation in 3DRA and CTA," Med. Image Anal., vol. 11, no. 3, pp. 224-241, 2007.

[29] G. Lathen, J. Jonasson, and M. Borga, "Blood vessel segmentation using multi-scale quadrature filtering," Pattern Recognit. Lett., vol. 31, pp. 762-767, Mar. 2010. 
[30] M. A. Palomera-Pérez, M. E. Martinez-Perez, H. Benítez-Pérez, and J. L. Ortega-Arjona, "Parallel multiscale feature extraction and region growing: Application in retinal blood vessel detection," IEEE Trans. Inf. Technol. Biomed., vol. 14, no. 2, pp. 500-506, Mar. 2010.

[31] Y. Zhao, Y. Liu, X. Wu, S. Harding, and Y. Zheng, "Retinal vessel segmentation: An efficient graph cut approach with retinex and local phase," PLoS ONE, vol. 10, p. e0122332, Apr. 2015.

[32] Y. Zhao, L. Rada, K. Chen, S. P. Harding, and Y. Zheng, "Automated vessel segmentation using infinite perimeter active contour model with hybrid region information with application to retinal images," IEEE Trans. Med. Imag., vol. 34, no. 9, pp. 1797-1807, Sep. 2015.

[33] M. Felsberg and G. Sommer, "The monogenic signal," IEEE Trans. Signal Process., vol. 49, no. 12, pp. 3136-3144, Dec. 2001.

[34] D. Boukerroui, J. Noble, and M. Brady, "On the choice of band-pass quadrature filters," J. Math. Imag. Vis., vol. 21, nos. 1-2, pp. 53-80, 2004.

[35] P. Kovesi, "Image features from phase congruency," Videre, London, U.K., Tech. Rep., 1995.

[36] I. Hacihaliloglu, A. Rasoulian, P. Abolmaesumi, and R. Rohling, "Local phase tensor features for $3 \mathrm{D}$ ultrasound to statistical shape+pose spine model registration," IEEE Trans. Med. Imag., vol. 33, no. 11, pp. 2167-2179, Nov. 2014.

[37] J. Orlando, E. Prokofyeva, and M. Blaschko, "A discriminatively trained fully connected conditional random field model for blood vessel segmentation in fundus images," IEEE Trans. Biomed. Eng., vol. 64, no. 1, pp. 16-27, Jan. 2017.

[38] A. P. Zijdenbos, B. M. Dawant, R. A. Margolin, and A. C. Palmer, "Morphometric analysis of white matter lesions in MR images: Method and validation," IEEE Trans. Image Process., vol. 13, no. 4, pp. 716-724, Dec. 1994.
[39] S. Roychowdhury, D. D. Koozekanani, and K. K. Parhi, "Iterative vessel segmentation of fundus images," IEEE Trans. Biomed. Eng., vol. 62, no. 7, pp. 1738-1749, Jul. 2015.

[40] J. Zhang, B. Dashtbozorg, E. Bekkers, P. Pluim, B. Duits, and R. Romeny, "Robust retinal vessel segmentation via locally adaptive derivative frames in orientation scores," IEEE Trans. Med. Imag., vol. 35, no. 12, pp. 2631-2644, Dec. 2016.

[41] Q. Li, B. Feng, L. Xie, P. Liang, H. Zhang, and T. Wang, "A crossmodality learning approach for vessel segmentation in retinal images," IEEE Trans. Med. Imag., vol. 35, no. 1, pp. 109-118, Jan. 2016.

[42] H. Bogunovic, J. Pozo, and A. Frangi, "Automated segmentation of cerebral vasculature with aneurysms in 3DRA and TOF-MRA using geodesic active regions: An evaluation study," Med. Phys., vol. 38, pp. 210-222, Apr. 2011.

[43] D. Pace, "Tubetk: An open-source toolkit of algorithms operating on images of tubes," in Proc. Int. Congr. Exhib. (CARS), vol. 7. 2012, pp. S79-S80.

[44] H. Bogunovic, J. M. Pozo, R. Cardenes, L. S. Roman, and A. F. Frangi, "Anatomical labeling of the circle of willis using maximum a posteriori probability estimation," IEEE Trans. Med. Imag., vol. 32, no. 9, pp. 1587-1599, Sep. 2013.

[45] G. Hamarneh and P. Jassi, "Vascusynth: Simulating vascular trees for generating volumetric image data with ground truth segmentation and tree analysis," Comput. Med. Imag. Graph., vol. 34, no. 8, pp. 605-616, 2010.

[46] P. Jassi and G. Hamarneh, "Vascusynth: Vascular tree synthesis software," Insight J., pp. 1-12, Jan./Jun. 2011.

[47] X. Cheng, Y. amd Hu, J. Wang, Y. Wang, and S. Tamura, "Accurate vessel segmentation with constrained b-snake," IEEE Trans. Imag. Process., vol. 24, no. 8, pp. 2440-2455, Aug. 2015. 
2017-09-26

\title{
Automatic 2-D/3-D vessel enhancement in multiple modality images using a weighted symmetry filter
}

\author{
Zhao, Yitian
}

IEEE

Zhao Y, Zheng Y, Liu Y., (2017) Automatic 2-D/3-D vessel enhancement in multiple modality images using a weighted symmetry filter, IEEE Transactions on Medical Imaging, Volume 37, Issue 2, February 2018, pp. 438-450

http://dx.doi.org/10.1109/TMI.2017.2756073

Downloaded from Cranfield Library Services E-Repository 